\title{
Percepción social de los maestros: análisis de la figura docente a través de Twitter
}

\author{
Ernesto Colomo Magaña* \\ Ángel Ignacio Aguilar Cuesta*
}

\section{Resumen}

En un contexto sociocultural dinámico y flexible, las funciones y competencias que se asocian a los docentes se van adaptando a las demandas y necesidades que se generan en la sociedad. Con el fin de conocer cómo es el maestro que la sociedad valora actualmente, este estudio analiza la figura del docente a través de la red social Twitter a partir del hashtag \#DíaMundialdelDocente. La muestra la componen 1441 tweets que fueron sometidos a un análisis en dos fases: una clasificación de los mensajes por temática y una posterior categorización en función de los arquetipos de maestros. Los resultados reflejan que la temática que más mensajes ha registrado ha sido la reivindicativa, mientras que el arquetipo más valorado a nivel social es el del maestro auténtico y personal. En las conclusiones se destaca el reconocimiento social positivo que existe sobre la figura del maestro, así como la posibilidad de incorporar las cualidades y competencias que son valoradas por la sociedad a los procesos de formación inicial y continua del profesorado.

Palabras clave

Características del maestro, rol del maestro, redes sociales, opinión pública, Twitter, influencia de la tecnología.

Recepción original: 15 de mayo de 2019

Aceptación: 04 de septiembre de 2019

Publicación: 20 de gener de 2020

\section{Introducción}

El progreso y evolución de la sociedad va unido a un desarrollo eficiente y positivo del campo educativo, convirtiéndose en una realidad que favorece mejoras a nivel personal y social. En este ámbito, los agentes participantes en dicho proceso se convierten en actores de todas aquellas transformaciones, cambios y mejoras ligadas a los procesos formativos. Por la responsabilidad y compromiso que adquiere en su función de transmitir el conocimiento y ayudar al desarrollo identitario de los estudiantes, los maestros se convierten en figuras claves a nivel social. Se trata de una profesión para la que hace falta algo más que el dominio de unos conocimientos o desarrollar unas destrezas, siendo la parte actitudinal, es decir, el comportamiento,

(*) Profesor adjunto del Área de Educación en la Universidad Internacional de Valencia. Doctor en Ciencias de la Educación, Licenciado en Pedagogía y Máster en Cambio Social y Profesiones Educativas por la Universidad de Málaga. Línea de investigación: educomunicación, formación del profesorado, figura docente y axiología. Dirección electrónica: ecolomomagana@gmail.com

${ }^{* *}$ Doctorando en Geografía por la Universidad Autónoma de Madrid y Personal Investigador en Formación por la misma universidad. Graduado en Historia y Máster Universitario en Formación del Profesorado por la Universidad de Málaga. Línea de investigación: TIC y educación, catastro histórico siglo XVIII y formación del profesorado. Dirección electrónica: angelignacioaguilar@gmail.com 
valores y actitudes que desarrolle e implemente el docente en el aula, un factor clave (Esteve, 2006). Por ello, se hace necesario que los maestros logren adquirir una serie de competencias profesionales y cualidades personales que son requeridas para un buen desempeño de la función docente. Todas ellas deben conformar parte de su ser, configurándose a nivel personal y profesional para dar lugar a su identidad.

En este sentido, se hace preciso aclarar que el concepto de identidad, con la consiguiente búsqueda personal y su evolución, ha sido un tema de interés continuo para el ser humano (Ruiz, 2005). Somos conscientes de que no estamos determinados al nacer (Merino, 2002), sino que serán nuestras vivencias y experiencias las que den sentido a nuestra vida y nos permitan progresar como seres humanos. Esta realidad nos obliga a un proceso continuo de reconstrucción personal, determinado también por el dinamismo y flexibilidad de la realidad, estando la identidad siempre abierta al cambio. Se constituye, por tanto, en una necesidad psíquica y social (Colomo y Domínguez, 2015) que favorece tanto a la madurez personal como a la convivencia pacífica y enriquecedora con el grupo social de referencia. Para ello, la forma en que interactuemos con la realidad y el significado con que dotemos a los diferentes hechos que vivenciemos serán claves para definir quiénes somos, tanto a nivel personal como profesional.

Si ponemos el foco en la identidad profesional docente (IPD), nos situamos ante un elemento que se genera y modifica al interaccionar en el contexto educativo (Rodríguez, González y Gutiérrez, 2019). La IPD se va generando a partir de las experiencias y las relaciones que se producen durante la formación inicial-continua y en el ejercicio de la labor docente. Está conformada por elementos personales (valores o compromiso para con la educación), cognitivos (nivel de conocimiento pedagógico y específico de la asignatura) y socioculturales (realidad política, multiculturalidad, etc.), de manera que la definición de quiénes somos cómo docentes encuentra respuesta en diferentes espacios educativos (Granados, Tapia y Fernández, 2017). En este proceso de conformación personal, los docentes se ven determinados por varios factores: por un lado, les influyen sus vivencias y experiencias en el contexto escolar; por otro lado, el escenario educativo en el que se desarrollan y con el que interactúan de forma constante. Ambos elementos son claves en el proceso de construcción de significados sobre su labor educativa, configurándose por este motivo identidades distintas y únicas (González y Fuentes, 2011). De esta manera, la identidad se va transformando y modificando en función de los cambios de la realidad personal y educativa, por lo que estamos ante un proceso continuo de (re)construcción (Correa, Fernández, Gutiérrez, Losada y Ochoa, 2015; Tello, 2016) que da lugar a que cada maestro sea único en la forma en que afronte el ejercicio docente (Lasky, 2005), aspecto que se ha convertido en un campo cada vez más estudiado (Akkerman y Meijer, 2011; Caballero y Bolívar, 2015; Pennington y Richards, 2016; Olven, 2016; Serret, Martí y Corbatón, 2016). En definitiva, la construcción de la identidad profesional se convierte en un proceso reflexivo para definir qué tipo de docente somos y cómo podemos seguir mejorando (Marcelo, 2010), de manera que seguirá evolucionando de forma constante para adaptarse a unas nuevas realidades que se originan en aras del desarrollo y avance de la sociedad. Es muy importante tener en consideración estas necesidades ya que son las que nos permitirán definir el tipo de docente que precisa la sociedad, debate que se ha convertido en habitual 
en diferentes foros y en donde no existe una respuesta unívoca ni sobre las competencias que debe tener ni respecto a qué tipología de docente es la más enriquecedora. Por ello, toca trasladar a la sociedad esta misma cuestión, para así tener una idea real sobre cómo es el maestro que se valora a nivel social.

Tenemos que partir de la realidad de que, a nivel social, la profesión docente está bien considerada (Pérez y Rodríguez, 2013), circunstancia que no impide que existan múltiples visiones y opiniones sobre la labor de los maestros. Se pretende conocer la percepción de los rasgos y características que más se valoran de los docentes para promoverlos o incorporarlos (en su defecto) en los planes formativos de los mismos. Para poder conocer la opinión latente en la sociedad, hemos recurrido a la visión que se traslada sobre esta realidad en los medios sociales. La razón es que estamos actualmente en una cibersociedad globalizada donde los diferentes canales comunicativos trasladan ideas que impactan e influyen en la conformación de los contextos, dando lugar así a nuevos espacios de interacción y relación (Gil de Zúñiga, Jung y Valenzuela, 2012; Loader, Vromer y Xenos, 2014). Entre las múltiples opciones, hemos seleccionado Twitter por convertirse en un vehículo comunicativo en tiempo real (Deller y Hallam, 2011), permitiendo recopilar mensajes sobre diferentes situaciones, hechos, reflexiones o debates con rapidez y una gran difusión. Esta red social ofrece la posibilidad de interactuar con límite en la extensión de los mensajes (240 caracteres), pudiendo establecer relación de seguidor o con menciones y retweets (Romero y Robinson, 2017). Un recurso habitual para las interacciones son los hashtags. Se trata de una funcionalidad que permite agrupar los mensajes de usuarios sobre un tema en concreto (Bruns y Burgess, 2012), favoreciendo así una participación activa en torno a debates y discusión sobre el tema de referencia.

Tomando como referencias otros estudios de análisis de datos sobre el contenido de los mensajes en Twitter dentro del área educativa (Davis, 2015; Motos y Aguilar, 2018), en este trabajo se recopilarán y analizarán los mensajes respecto al hashtag \#DíaMundialDelDocente, obteniendo así los mensajes que se generaron sobre esta efeméride en dicha red social. El Día Mundial del Docente nace como una iniciativa impulsada por la UNESCO y la colaboración de UNICEF, el PNUD, la Organización Internacional del Trabajo (OIT) y la Internacional de la Educación. Se celebra cada 5 de octubre desde 1994 y pretende visibilizar la importancia de la figura del maestro, aprovechando este homenaje a nivel global para agradecer y valorar su labor educativa, generando así a nivel social un reconocimiento y respeto hacia el ejercicio docente por la relevancia de su papel en el progreso social. Además, es un contexto que permite recordar el trabajo y esfuerzo de todos aquellos educadores que lucharon por lograr alcanzar una mejor educación, potenciando aspectos como la calidad, la universalidad, la igualdad o la equidad en la misma. En el año 2018, el seleccionado para nuestro estudio, la temática se centró en conmemorar el $70^{\circ}$ aniversario de la Declaración Universal de los Derechos Humanos, entre cuyos derechos fundamentales se recoge el de la educación. Por todo ello, la relevancia e impacto registrados en Twitter sobre los docentes en esta efeméride serán la base de nuestra investigación. 
Por consiguiente, este estudio pretende conocer la percepción social que hay de los docentes mediante el análisis de las interacciones en el hashtag \#DíaMundialDelDocente. El objetivo general se divide en varios objetivos específicos, entre los que se destacan: a) conocer las características que se valoran de los maestros a través de los tweets analizados; b) vincular las características con las diferentes tipologías de maestros; c) profundizar en los rasgos más habituales de cada arquetipo.

\section{Material y métodos}

Con el fin de conocer cuál era la visión social que se trasladaba sobre los maestros a través de las redes sociales, se seleccionó Twitter para realizar el estudio por su potencial y permeabilidad. Una vez elegida dicha red, fue preciso crear un script que permitiera recolectar mediante la API Rest todos los tweets que se publicaron con el hashtag \#DíaMundialDelDocente (incluidos además los del \#DiaMundialDelDocente, por las habituales faltas de ortografías en redes sociales). Esta acción, nos permite recuperar información sobre cualquier fenómeno o temática dentro de un marco temporal definido, permitiéndonos infinidad de análisis y comparativas sobre diferentes hechos o la evolución de una misma realidad en el tiempo. En nuestro caso, el foco lo queríamos situar en el homenaje al maestro a nivel global en el año 2018, por lo que fue necesario delimitar también las fechas de dicha recolección al día concreto de la celebración, ya que la repercusión en Twitter en torno a esta efeméride también produjo interacciones en los días previos y posteriores.

Todo este proceso nos ayudó a la obtención de un total de 1441 tweets durante el día 5 de octubre de 2018, aunque en días anteriores y posteriores también hubo actividad, como hemos comentado, pero al no estar dentro de la efeméride quedaron fuera de este estudio.

\section{Figura 1. Elementos del script creado para la extracción de tweets}

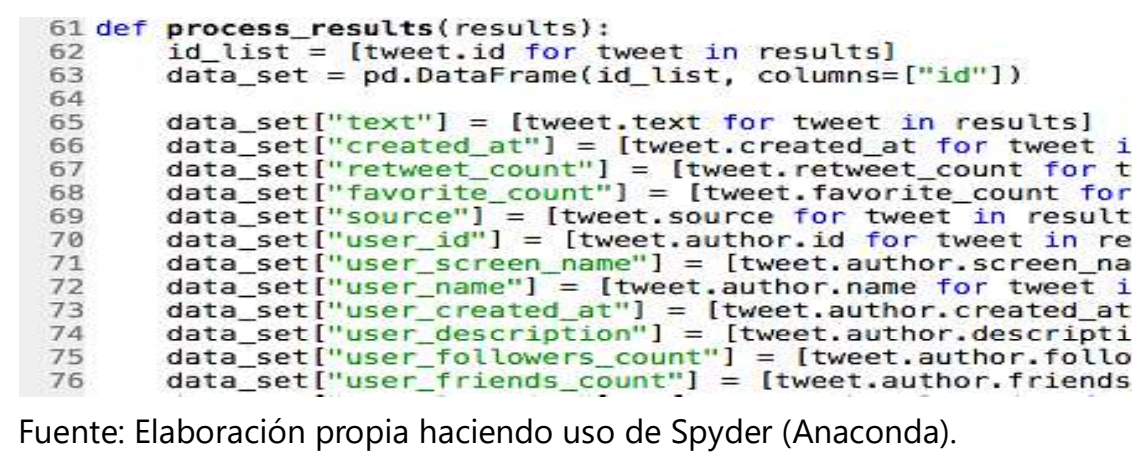

Para el análisis de contenido de los tweets recolectados y categorizar la información, se han utilizado cuatro tipologías de maestros diferenciadas por los rasgos y actitudes que las componen. Partimos de la propuesta inicial de Esteban y Mellen (2016), quienes distinguían las tipologías del maestro auténtico y personal, el maestro transmisor de historias y el maestro motivador del aprendizaje, a la cual incorporamos la del maestro como agente del cambio social. Pasamos a conceptualizar cada una de ellas.

El primer tipo de maestro es el auténtico y personal. El factor que mejor define al mismo es la empatía y la relación personal que crea con el estudiante, buscando tanto el cuidado (Noddings, 2010) como el perfeccionamiento mutuo (Scott, 2000). 
En este sentido, el docente pone todo su énfasis en ayudar al desarrollo y evolución del alumnado, atendiendo a las diferentes demandas y necesidades que puedan precisar. No obstante, también cuida su crecimiento profesional y personal mediante la labor docente, cuidando aspectos como su carácter, su escala de valores o su formación ético-moral (Matsuba, Murzyn y Hart, 2011). Trabajar de esta forma en su (re)construcción identitaria les permite un mayor control y gestión emocional (Buitrago y Cárdenas, 2017) tanto para tomar decisiones más justas en el ejercicio docente como para ayudar al alumnado a que reconozcan y regulen sus emociones (Aguaded y Pantoja, 2015). Estamos, por tanto, ante un docente comprometido con el desarrollo integral tanto de sus estudiantes como de sí mismo.

El siguiente arquetipo es el del maestro transmisor de historias. Son docentes que se definen por la pasión y el deseo de saber sobre los contenidos de su disciplina (Asún, Zúñiga y Ayala, 2013; Flores, Yedaide y Porta, 2013; Recalcati, 2016). Esta mezcla de inquietud y motivación es un factor clave que les facilita progresar en la adquisición de conocimientos sobre su área (López, 2016), haciendo más interesantes sus lecciones. Son docentes con una amplia formación cultural, aspecto que les permite establecer vínculos significativos entre los contenidos de su disciplina y lo que acontece en el mundo real (Nussbaum, 2001), logrando así una formación más global y holística. Es la preferencia y amor hacia el tema de su asignatura y como lo extrapola a sus clases lo que genera atracción en el estudiante (Delpit, 2003), logrando que el mismo se sienta motivado hacia dichos contenidos (Buxarrais, Vilafranca y Bujons, 2016; Ordine, 2013). En definitiva, es un docente que enriquece sus lecciones con contenidos afines y las traslada de forma apasionada y motivadora para el estudiante.

A continuación, encontramos la tipología del maestro motivador para el aprendizaje. Se caracteriza por poseer un profundo desarrollo de las competencias pedagógicas, garantizando así afrontar el hecho educativo de forma exitosa. En este sentido, sus habilidades y destrezas le permiten controlar y gestionar de forma adecuada los procesos educativos (Martín, Conde y Mayor, 2014), incorporando y aplicando diferentes metodologías, técnicas docentes y estrategias didácticas en la realidad del aula para alcanzar procesos de enseñanza-aprendizaje eficaces y de calidad (Applegate, 2010), todo ello sin perder de vista la necesidad de estar en continuo aprendizaje para adaptarse a los cambios e innovaciones que requiere la educación del futuro. La finalidad es que instauren en los estudiantes el deseo y la motivación por aprender al dar un papel protagonista a elementos más creativos y divertidos ligados al proceso formativo (Cabero y Marín, 2017; Centeno, Alcaraz, González, Oropeza y Ramírez, 2017; Quevedo y Arruti, 2018). Estamos ante un maestro que domina los escenarios y recursos para implementar procesos óptimos de aprendizaje.

Por último, tenemos el arquetipo del maestro como agente de cambio social. Se caracterizan por entender a la educación como un ejercicio que favorece una transformación enriquecedora del estudiante y del contexto social en el que se inserta (Freire, 2005). De este modo, el acto educativo es concebido como una posibilidad real de cambio, dirigiendo la misma al logro del bienestar social (Rodríguez, González y Gutiérrez, 2019), con el fin de alcanzar una realidad mejor en la actualidad y para el futuro (Romero y Núñez, 2017). Este tipo de docentes suelen sentir una 
atracción por la enseñanza, entendiendo que su labor formativa trasciende el aula y se dirige a un trabajo en aras de mejorar la realidad social (Badia, Monereo y Meneses, 2013).

\section{Resultados}

La elección de la efeméride sobre el Día Mundial del Docente y su impacto en la red social Twitter ha conllevado el recopilar un total de 1441 tweets en los que se aborda el papel y figura del docente. Utilizamos el hashtag \#DíaMundialdelDocente (y \#DiaMundialdelDocente sin tilde) durante la fecha de celebración del homenaje a los docentes (5 de octubre de 2018), cuya repercusión se elevó a los 6.760 si tenemos en consideración los retweets y favoritos que obtuvieron los mensajes difundidos, dando así lugar a un espacio amplio de debate y reflexión sobre cuestiones relevantes del ámbito educativo. Comenzamos realizando una primera fase de categorización en función de la tipología de los tweets, entre las que encontramos reivindicación, homenaje, informativo, otros y características del buen docente (ver tabla 1).

\section{Tabla 1. Número de tweets emitidos según su tipología}

\begin{tabular}{ccc}
\hline Tipología & No de tweets & $\%$ \\
\hline Reivindicación & 440 & $30,53 \%$ \\
Homenaje & 302 & $20,96 \%$ \\
Informativo & 209 & $14,50 \%$ \\
Otros & 92 & $6,39 \%$ \\
Características del buen docente & 398 & $27,62 \%$ \\
Total & $\mathbf{1 4 4 1}$ & $\mathbf{1 0 0 \%}$ \\
\hline
\end{tabular}

Fuente: Elaboración propia a partir de los tweets extraídos.

La primera categoría es la de reivindicación, con un 30,53\% del total de mensajes. Se trata de tweets que, aprovechando la efeméride y desde un enfoque positivo, trasladan diferentes demandas y necesidades de la realidad docente en la actualidad, convirtiéndose Twitter en un vehículo comunicativo de calado social. El foco se sitúa principalmente en las condiciones en las que se ejerce el magisterio en las aulas, elevando a los poderes políticos las necesidades que primar para mejorar la labor educativa. De esta manera, aparecen mensajes reclamando una mejora sustancial de las condiciones laborales, centrando las mismas en disminuir los trámites burocráticos que hace un docente, las ratios de alumno por aula y mayor agilidad para cubrir las plazas vacías en los centros escolares. Junto a ello, se enarbola la bandera de la formación inicial y continua como elemento clave para mejorar la calidad y eficacia de los procesos de enseñanza-aprendizaje.

La categoría homenaje también ha recopilado gran cantidad de tweets, con un $20,96 \%$ del total. En ella confluyen mensajes de corte personal y social, aprovechando este medio social para reforzar la imagen del docente y su labor para con la 
sociedad. En este sentido, los mensajes extrapolan vivencias personales y experiencias significativas, lo que conlleva tweets con una carga emocional relevante que ha de ser considerada.

En lo que respecta a los mensajes categorizados como informativos, han supuesto un 14,50\% del total. En ellos se han recogido noticias en torno a la efeméride del Día Mundial del Docente y al papel que la UNESCO tiene en este homenaje, abordando los orígenes de la creación de este día, así como la temática que se trabajaba de forma específica en este año 2018.

En cuanto a la tipología de otros, han sido categorizados en ella el 6,39\% de los tweets. No olvidemos que los hashtags permiten interactuar a los usuarios de forma libre sobre diferentes temáticas sin que ello conlleve una obligación de que el contenido de su mensaje verse sobre la misma. Esta situación ha dado lugar a que aparezcan mensajes con diferentes temáticas. Destacan el humor, con tintes tanto positivos como negativos; y cuestiones personales narradas en primera persona sobre experiencias de corte negativo sufridas en el contexto escolar.

Mención especial para nuestro trabajo tiene la tipología característica del buen docente, donde se han recogido mensajes en los que se abordan los rasgos que definen a los distintos tipos de docentes, con un 27,62\%. Partiendo de la propuesta de arquetipos de maestros trabajada por Esteban y Mellen (2016), hemos realizado una segunda fase de categorización, trabajando exclusivamente sobre el $27,62 \%$ de la muestra original. En este sentido, de los 398 tweets analizados, solo el $6 \%$ no ha sido categorizado en ninguna de las tipologías de maestros al no poder relacionar su contenido con los rasgos definitorios de los tipos de docentes (ver tabla 2).

Tabla 2. Número de tweets asociados a los arquetipos de docentes

\begin{tabular}{ccc}
\hline Tipología & No de tweets & $\%$ \\
\hline Maestro auténtico y personal & 290 & $72,9 \%$ \\
Maestro como agente de cambio social & 46 & $11,6 \%$ \\
Maestro motivador para el aprendizaje & 20 & $5 \%$ \\
Maestro transmisor de historias & 18 & $4,5 \%$ \\
No procede & 24 & $6 \%$ \\
Total & 398 & 100 \\
\hline
\end{tabular}

Fuente: Elaboración propia a partir de los tweets extraídos.

Para el análisis cualitativo pormenorizado de los mensajes que formaban parte de la categoría características de un buen docente, partiremos de los arquetipos docentes definidos en el apartado de material y métodos. Por los porcentajes registrados, la relevancia y espacio de análisis se adecua al impacto de estas tipologías en la red social Twitter a través del hashtag \#DíaMundialDelDocente, estableciendo subapartados para abordar por separado cada uno de los tipos de maestros. De cara a la lectura, se hace preciso incidir en que la polaridad de los mensajes tiene 
una tendencia positiva natural, ya que hemos partido de una efeméride en la que se homenajea a los maestros. Además, la mayoría de los mensajes emitidos por los usuarios son fruto de sus experiencias educativas durante su paso por las distintas etapas formativas, de manera que los recuerdos que se han recopilado han tenido una connotación afectuosa en su mayoría. Pasamos a analizar la visión social de las diferentes tipologías de maestros.

\section{Maestro auténtico y personal}

El arquetipo que más mensajes ha registrado es el del maestro auténtico y personal con un $72,9 \%$ de los tweets. Se trata de un perfil de docente caracterizado por los vínculos afectivos que genera con el alumnado en el proceso de enseñanza-aprendizaje. En este sentido, cobra especial relevancia una relación docente-estudiante sustentada en el cariño, lo que les genera a los segundos un recuerdo sobre su etapa formativa que será clave en su proceso de construcción identitaria (@EugeniaRomeraAL: https://twitter.com/EugeniaRomeroAL/status/1048174987545174017; @semisegorbe: https://twitter.com/semisegorbe/status/1048305495335944196). Y es que los docentes se convierten en figuras claves para el alumnado a nivel social, siendo ejemplo de valores y comportamientos (Sanderse, 2013), que pueden ser imitados y asumidos por el alumnado o rechazados, dependiendo ya de la conjunción de dichas realidades con su propia personalidad y escala de valores. No obstante, es interesante que las actitudes ético-morales que los profesores materialicen en clase sean positivas y favorezcan el enriquecimiento identitario de estos. Al fin y al cabo, nos encontramos ante una relación bidireccional donde los docentes se preocupan por lo que el alumno fue (partir de una visión neutral del alumno, evitando encasillarlo o etiquetarlo, respetando así sus experiencias y vivencias que le han hecho ser quien es en el momento actual), es (trabajo diario compartiendo procesos formativos no solo a nivel cognitivo, sino también social y afectivo, claves todos para un proceso de reconstrucción identitaria continua) y será (sentar las bases y pilares para que el alumno pueda desarrollar todo su potencial y tenga las herramientas y mecanismos para enfrentarse a los diferentes problemas que pueda deparar la realidad), actuando como guía en el proceso de enseñanza-aprendizaje y como acompañante durante su evolución y desarrollo identitario. Se trata de cuidar todo aquello que acontece en el escenario educativo, siendo para ello clave la figura de docentes que priorizan el factor humano (Esteban 2018; Esteve, 2006), como trasciende en el tweet de @ApadrinaCiencia (https://twitter.com/ApadrinaCiencia/status/1048130359186141184). En definitiva, se trata de que los docentes sean conscientes que su labor e influencia no se puede calcular, pues influye en muchas generaciones a nivel intelectual y a nivel personal (Adams, 1951).

Otro aspecto a considerar es el papel que la vocación juega en este tipo de maestros. Partiendo de que su preocupación reside tanto en la formación de su alumnado (Ibáñez, 2017) como en su desarrollo y crecimiento personal continuo (Bermúdez y Laspalas, 2017) este elemento se convierte en un factor clave para la identidad docente (Esteve, 2009) para enfrentarse a las dificultades que conlleva su labor y también a las alegrías de desempeñar esta profesión. Se recopilan tweets considerando tanto una visión de pasión hacia el ejercicio docente (@garozsclass: https://twitter.com/garozsclass/status/1048201248862863361) como una actitud 
de compromiso hacia las funciones que quedan ligadas a su labor (@AndresLTrujillo: https://twitter.com/AndresLTrujillo/status/1048181866568843264). No obstante, solo con amar lo que se hace no es suficiente para garantizar procesos de enseñanza de calidad, siendo preciso que los docentes adquieran las competencias, destrezas y habilidades necesarias para poder desarrollar su trabajo de forma exitosa (Larrosa, 2010). Son estas características otra de las temáticas que más mensajes han recopilado por el debate social que se genera sobre una cuestión que puede convertirse en interminable por los múltiples enfoques y perspectivas que pueden aportar propuestas a este tema. En su estudio, Timmerman (2009) centró su esfuerzo en recopilar diferentes actitudes y aptitudes que son necesarias desarrollar para la labor docente, siendo interesante que estén implementadas en los programas de formación inicial y continua, coincidiendo muchas de ellas con las recogidas en algunos de los mensajes recopilados (@javinsan: https://twitter.com/javinsan/status/10481531000103 15776; @LiSabater: https://twitter.com/LiSabater/status/1048096213478907904). Como podemos observar, el impacto que este tipo de docente tiene en las experiencias y vivencias de los usuarios que han interaccionando en torno al hashtag analizado, nos traslada la idea de la relevancia de promover los rasgos del maestro auténtico y personal en todo programa formativo tanto para futuros docentes como en ejercicio.

\section{Maestro como agente de cambio social}

La tipología del maestro como agente de cambio social registró un $11,6 \%$ de los tweets dentro de la supracategoría características del buen docente. En este arquetipo, el docente se entrega a su alumnado, centrando sus esfuerzos en el crecimiento y desarrollo evolutivo de los discentes para conformar personas autónomas y comprometidas con la sociedad presente y futura. De este modo, el proceso formativo se constituye como un factor clave para el progreso y evolución de la humanidad (@irimarq: https://twitter.com/irimarq/status/1048320349857882112), teniendo la labor docente una vocación de servicio y de darnos al estudiante (Álvarez, Martínez, González y Buenestado, 2017). Se trata de una labor que pone el foco en que los estudiantes adquieran los mecanismos y destrezas necesarias para ser autónomos. Para ello, es fundamental que los profesores crean en las posibilidades y potencialidades de los mismos (Barquín, Alzola y Madinabeita, 2012) y que eviten la resignación ante las continuas trabas y dificultades con las que se encuentran en el ámbito educativo. En este sentido, el hashtag se inundó de frases famosas y motivadoras que enarbolan el papel de la educación en la construcción social, como la mítica sentencia de Nelson Mandela (@CsGranCanaria: https://twitter.com/CsGranCanaria/status/1048133276911788032). No olvidemos que el ejercicio docente es la semilla que origina al resto de las profesiones, siendo así clave el contexto educativo en el progreso social (@_Raquel_Morales: https://twitter.com/_Raquel_Morales/status/1048151126229618688). Para finalizar, destacar en esta tipología de maestro la idea de que los docentes son responsables de que el alumnado aprenda a pensar por sí mismo (Kong, 2014), con la importancia que este hecho conlleva para la toma de decisiones y defensa de sus derechos a nivel global (@mjrr_spain: https://twitter.com/ mjrr_spain/status/1048144153220136960; @CsSanSe: https://twitter.com/ CsSanSe/status/1048257320826929152). De este modo, los maestros se convierten 
en inspiradores y promotores del cambio que deben llevar a cabo las nuevas generaciones que llenan las aulas en la actualidad.

\section{Maestro motivador para el aprendizaje}

El maestro motivador para el aprendizaje alcanza el 5\% de los tweets en la supracategoría trabajada. Se convierten en facilitadores del proceso de enseñanza-aprendizaje, aportando sus conocimientos respecto a metodologías, estrategias didácticas y diseño de procesos pedagógicos adecuados a las características y necesidades de cada alumno de forma personal (García Hoz, 1986; Mojarro, Rodrigo y Etchegaray, 2015), sin perder de vista el grupo-clase. Se trata de facilitar experiencias de aprendizaje que lleven a los discentes a ser más críticos, reflexivos y autónomos (@Mexicanos1o: https://twitter.com/Mexicanos1o/status/1048325456380841984). $Y$ es que el conocimiento de la función y ejercicio docente dota de calidad y eficiencia a los procesos formativos, aspecto que no podría alcanzarse de no dominar los entresijos del acto educativo. Todo ello, es fruto de un compromiso que adquiere de forma consciente el educador, siendo sabedor de que los procesos innovadores y atractivos (González y Rodríguez, 2014), requieren de un esfuerzo continuo, ya que como dice @almudenixx (https://twitter.com/almudenixx/status/1048189434615803905), «dejan su tiempo y esfuerzo en crear clases atractivas para que su alumnado disfrute aprendiendo».

\section{Maestro transmisor de historias}

El arquetipo que ha registrado menor número de menciones es el del maestro transmisor de historias, registrando solo un 4,5\% de tweets dentro de la supracategoría de las características del buen docente. Se encargan de despertar la curiosidad (Dapía, 2008; Mora, 2013), de avivar el fuego del saber por la forma en que trasladan los contenidos y enriquecen sus lecciones. Nos retomamos a la figura icónica de los contadores de historias, de forma que los docentes atraen al alumnado hacia el conocimiento sobre el eje narrativo que construye en torno a los contenidos. De esta manera, en las clases se vivencian los conocimientos, favoreciendo una experiencia pedagógica que permite aprender disfrutando (@pedro_casares: https://twitter.com/pedro_casares/status/1048226877813 522433). Encontramos así a un profesor que mezcla tres grandes pasiones: enseñar, aprender y su materia (@Pepmari4: https://twitter.com/pepmari4/status/104817 0387870240769). Aquí podemos ver una sinergia cíclica en dichos elementos, donde el docente ama el ejercicio docente de compartir sus conocimientos con el alumnado, para lo cual debe seguir actualizándose de manera continua y adquiriendo nuevos conocimientos que enriquezcan su materia, ese campo de contenidos y conocimientos cuya transmisión lo acercó a la labor educativa. En definitiva, es mediante el arte de la palabra y la oratoria como los docentes llegan al alumnado para despertar su interés y transmitirle los conocimientos.

\section{Discusión y conclusiones}

Este trabajo pretendía conocer la percepción que existe a nivel social sobre la figura del docente. Es importante reseñar que la sociedad tiene asumidas ciertas creencias respecto al fenómeno educativo, dando lugar a prejuicios y verdades no refutadas 
que determinan la visión y opinión sobre dicha realidad. En esta línea, los maestros como agentes protagonistas y responsables de garantizar procesos de enseñanzaaprendizaje de calidad y eficientes, llevan también vinculadas ciertas etiquetas que cuestionan el nivel formativo, la complejidad/dificultad del desempeño de su labor y las condiciones de trabajo (UNESCO, 2018). Sin embargo, en el análisis realizado en nuestra muestra se traslada una percepción positiva sobre los docentes, no debiéndose en exclusiva a que la muestra se haya seleccionado en torno a una efeméride sobre la figura del maestro, sino que existe apoyo social respecto a su importancia y conciencia de la necesidad de alcanzar mejoras para un ejercicio educativo de calidad (Valle, 2013). En las categorías de homenaje, características del buen docente $y$, también, en la de reivindicaciones se han registrado mensajes de reconocimiento hacia competencias y cualidades relacionadas con una visión humanista de la docencia, destacando el compromiso con el quehacer educativo y con una pedagogía sustentada en el amor. Respecto a la categoría informativa, la misma ha servido como vehículo comunicativo para trasladar datos y detalles sobre el origen y sentido de la efeméride sin otra atribución en particular.

Pasando a comentar de forma detallada los objetivos específicos, en primer lugar, queríamos conocer cuáles eran las características que a nivel social más se valoran de los docentes. Los análisis nos permiten tener una percepción general sobre este aspecto, dándonos así lugar a procesos de reflexión en torno a la configuración y desarrollo de los procesos formativos de los docentes. Es importante subrayar que el origen de los mensajes son los recuerdos y experiencias vividas por los tweeteros en su etapa escolar, de manera que la concepción sobre la figura ideal de docente estará mediada por los profesionales de la educación que han pasado por sus vidas (Madrid y Mayorga, 2017). Teniendo como base una efeméride que homenajea la figura del docente, el foco no se sitúa en sus competencias pedagógicas o los profundos conocimientos que tenían sobre la materia, sino que lo que se destaca es el factor humano respecto a su labor docente, es decir, su forma de estar en el mundo como docente.

En el ejercicio de relacionar los principales rasgos y características recogidos en los tweets respecto a los arquetipos docentes, los datos siguen en la misma línea de subrayar la importancia del componente empático del profesorado. La tipología más valorada es la del maestro auténtico y personal en la que predomina el cultivo de las relaciones personales y la transmisión de valores y comportamientos éticos y morales adecuados, coincidiendo con la investigación de Pérez y Rodríguez (2013). Los porcentajes del resto de tipologías han sido significativamente inferiores, por lo que, como hemos comentado, se valora mucho más al docente por su forma de ser y actuar como persona que por su interés en transformar la sociedad, el profundo dominio de su materia o la competencia pedagógica para garantizar el aprendizaje de forma satisfactoria. No obstante, están presentes porque sus rasgos son relevantes también para los procesos formativos. Si nos centramos con más detalle en los rasgos que han obtenido mayor frecuencia en los diferentes arquetipos, predomina los relacionados con la categoría del maestro auténtico y personal por su masivo registro. De esta manera, aspectos como la vocación, la empatía, el cariño y el cuidar de forma positiva las relaciones entre docentes y estudiantes han tenido un alto impacto, asemejándose a las cualidades destacadas en otros estudios que analizan la figura del maestro que deja huella (Esteban, 2016; Martínez, Esteban, Gonzalo y 
Payá, 2016). De las otras tres tipologías, pese a tener un menor registro, podemos destacar la pasión por el conocimiento, la preocupación por el bienestar social o el desarrollo de propuestas educativas innovadoras.

Toda esta información nos debe poder permitir llevar a cabo un profundo proceso de reflexión sobre si existe relación entre el tipo de docente que valora la sociedad y cómo es el prototipo de profesor que preparamos para la labor educativa a tenor de los currículos y planes de estudios implementados en la formación inicial y continua. Con ello no queremos decir que la visión social cuestione los tratados pedagógicos que han consolidado las bases de la formación de los maestros, pero sí que es preciso entender cuál es la realidad de nuestra sociedad actual y qué demanda esta de los docentes, para comprobar si estamos promoviendo y potenciando algunos de esos aspectos que pueden resultar muy beneficiosos 0 , por el contrario, no están siendo considerados. En este sentido, se deben centrar los esfuerzos en la formación del carácter de los maestros por su importancia de cara a ser ejemplo de valores y comportamientos para los educandos (Esteban, 2018; Matsuba, Murzyn y Hart, 2011), junto con todas aquellas competencias que permitan enfrentarse con éxito a los retos actuales de la educación.

La profesión docente, como hemos podido ver, sigue jugando un papel clave a nivel social, siendo agitadores para el pensamiento crítico y representantes de la confianza en la bondad y mejora continua del ser humano. Como expusiera Unamuno (1917), nos definimos como maestros y vivimos en un continuo pensar y sentir y hacer pensar y sentir, siendo este el principal fin de nuestra labor.

\section{Referencias}

Adams, H. (1951) La educación de Henry Adams. México, Ediciones Botas.

Aguaded, M.C. y Pantoja, M.J. (2015) «Innovar desde un proyecto educativo de inteligencia emocional en primaria e infantil». Tendencias Pedagógicas (Madrid, Universidad Autónoma de Madrid), 26, pp. 69-88. doi: http://dx.doi.org/10.15366/tp2015.26

Akkerman, S.F. y Meijer, P.C. (2011) «A dialogical approach to conceptualizing teacher identity». Teaching and Teacher Education (Ámsterdam, Elsevier B.V.), 27(2), pp. 308-319. doi: https://doi.org/10.1016/j.tate.2010.08.013

Álvarez, J.L., Martínez, M.J., González, H. y Buenestado, M. (2017) «El aprendizajeservicio en la formación del profesorado de las universidades españolas». Revista Española de Pedagogía (La Rioja, Universidad Internacional de La Rioja), 75(267), pp. 199-217. doi: https://doi.org/10.22550/REP75-2-2017-02

Applegate, J.R. (2010) «Teaching competencies and the Teacher Preparation Program». Improving College and University Teaching (United Kingdom, Taylor \& Francis, Ltd.), 25(4), pp. 226-230.

Asún, R., Zúñiga, C. y Ayala, M.C. (2013) «La formación por competencias y los estudiantes: confluencias y divergencias en la construcción del docente ideal». Calidad en la educación (Santiago de Chile, Consejo Nacional de Educación), 38, pp. 277-304. doi: https://doi.org/10.4067/S0718-45652013000100008

Badia, A., Monereo, C. y Meneses, J. (2013) «Affective dimension of university professors about their teaching: An exploration through the semantic differential technique». Universitas Psychologica (Bogotá, Pontificia Universidad Javariana), 13(1), pp. 161-173. doi: https://doi.org/10.11144/Javeriana.UPSY13-1.adup. 
Barquín, A., Alzola, N. y Madinabeita, M. (2012) «Innovación curricular en los grados de maestro en la facultad HUHEZI (Mondragon Unibertsitatea): la materia Educación intercultural y educación en valores». REDU. Revista de Docencia Universitaria (Valencia, Universitat Politècnica de València), 10(3), pp. 171-194.

Bermúdez, J.J. y Laspalas, F.J. (2017) «El profesor universitario: integración entre lo personal y lo profesional». Teoría de la Educación. Revista interuniversitaria (Salamanca, Universidad de Salamanca), 29(2), pp. 109-126.

doi: http://dx.doi.org/10.14201/teoredu292109126

Bruns, A. y Burgess, J. (2012) «Researching news discussion on Twitter: New methodologies». Journalism Studies (United Kingdom, Taylor \& Francis, Ltd.), 13(5-6), pp. 801-814.

Buitrago, R.E. y Cárdenas, R.N. (2017) «Emotions and Teachers' Professional Identity: Relations and Incidence». Praxis \& Saber (Boyacá (Colombia), Universidad Pedagógica y Tecnológica de Colombia), 8(17), pp. 225-247. doi: http://dx.doi.org/10.19053/22160159.v8.n17.2018.7208.

Buxarrais, M.R., Vilafranca, I. y Bujons, C. (2016) «Los contenidos en la formación de maestros y su incidencia en el carácter». En Esteban, F. [ed.]. La formación del carácter de los maestros. Barcelona, Edicions de la Universitat de Barcelona, pp. 73-88.

Caballero, K. y Bolívar A. (2015) «El profesorado universitario como docente: hacia una identidad profesional que integre docencia e investigación». REDU. Revista de Docencia Universitaria (Valencia, Universitat Politècnica de València), 13(1), pp. 57-77.

Cabero, J. y Marín, V. (2017) «La educación formal de los formadores de la era digital - los educadores del siglo XX|». Notandum (Oporto, Editora Mandruvá), 44-45, pp. 29-42. doi: http://dx.doi.org/10.4025/notandum.44.4

Centeno, G., Alcaraz, A.L., González, O.R., Oropeza, K.S. y Ramírez, G. (2017) «El lenguaje, una herramienta para aprender mediante el modelo de gestión Aprendizaje Invertido, un estudio de caso en alumnos de $5^{\circ}$ de primaria». Edutec. Revista Electrónica De Tecnología Educativa (Palma de Mallorca, Asociación para el desarrollo de la tecnología educativa y de las nuevas tecnologías aplicadas a la educación), 59, pp. 1-17. doi: https://doi.org/10.21556/edutec.2017.59.756

Colomo, E. y Domínguez, R. (2015) «Definiendo identidades: el "canciograma" como herramienta metodológica de autoconocimiento». REICE. Revista Iberoamericana sobre Calidad, Eficacia y Cambio en Educación (Madrid, Universidad Autónoma de Madrid y Red Iberoamericana de Investigación sobre Cambio y Eficacia escolar [RINACE]), 13(2), pp. 131-146.

Correa, J.M., Fernández, L., Gutiérrez, A., Losada, D. y Ochoa, B. (2015) «Formación del Profesorado, Tecnología Educativa e Identidad Docente Digital». RELATEC. Revista Latinoamericana de Tecnología Educativa (Cáceres, Universidad de Extremadura), 14(1), pp. 45-56. doi: https://doi.org/10.17398/1695-288X.14.1.45

Dapía, A. (2008) «Deconstrucción de la didáctica racionalista en el contexto de la formación docente. Hacia una didáctica constructivista». Revista Iberoamericana de Educación (Madrid, Organización de Estados Iberoamericanos), 45, pp. 3-25.

Davis, K. (2015) «Teachers' perceptions of Twitter for professional development». Disability and Rehabilitation (United Kingdom, Taylor \& Francis, Ltd.), 37(17), pp. 1551-1558. doi: https://doi.org/10.3109/09638288.2015.1052576

Deller, R. y Hallam, S. (2011) «Twitteringon: Audience research and participation using Twitter». Participations. Journal of Audience \& Reception Studies (Newcastle, Northumbria University), 8, pp. 216-245. 
Delpit, L. (2003) «Educators as "seed people" growing a new future». Educational Researcher (New York, SAGE Publishing), 32(7), pp. 14-21.

Esteban, F. [ed]. (2016) La formación del carácter de los maestros. Barcelona, Edicions de la Universitat de Barcelona.

Esteban, F. (2018) Ética del profesorado. Barcelona, Herder.

Esteban, F. y Mellen, T. (2016) «¿Por qué quieres ser maestro? ¿Cómo es un buen maestro? Ideas para la formación universitaria». Bordón (Madrid, Sociedad Española de Pedagogía), 68(2), pp. 185-198.

doi: https://doi.org/10.13042/Bordon.2016.68212

Esteve, J.M. (2006) «Las emociones en el ejercicio práctico de la docencia». Revista Teoría de la educación (Salamanca, Universidad de Salamanca), 18, pp. 85-107.

Esteve, J.M. (2009) «La formación de profesores: bases teóricas para el desarrollo de programas de formación inicial». Revista de Educación (Madrid, Ministerio de Educación, Cultura y Deporte), 350, pp. 15-29.

Flores, G., Yedaide, M.M. y Porta, L. (2013) «Grandes maestros: intimidad entre la educación y la vida. Pasión por enseñar en el aula universitaria». Revista de Educación (Mar de Plata, Universidad Nacional de Mar de Plata), 4(5), pp. 173188.

Freire, P. (2005) Pedagogía de la esperanza. México, Siglo XXI Editores.

García Hoz, V. (1986) La práctica de la educación personalizada. Madrid, Rialp.

Gil de Zúñiga, H., Jung., N. y Valenzuela, S. (2012) «Social media use for news and individual's social capital, civic engagement and political participation». Journal of Computer-Mediated Communication (New Jersey, John Wiley \& Sons, Ltd.), 17(3), pp. 319-336. doi: https://doi.org/10.1111/j.1083-6101.2012.01574.x

González, M. y Fuentes, E.J. (2011) «El Practicum en el aprendizaje de la profesión docente». Revista de Educación (Madrid, Ministerio de Educación, Cultura y Deporte), 354, pp. 47-70.

González, M. y Rodríguez, B. (2014) «La formación inicial de los profesores de lengua extranjera: un espacio para generar estilos de actuación». Bordón (Madrid, Sociedad Española de Pedagogía), 66(4), pp. 69-86. doi: https://doi.org/10.13042/Bordon.2014.66406

Granados, J., Tapia, A.M. y Fernández, J. (2017) «La construcción de la identidad de los docentes noveles: un análisis desde las teorías apriorísticas». REDU. Revista de Docencia Universitaria (Valencia, Universitat Politècnica de València), 15(2), pp. 163-178. doi: https://doi.org/10.4995/redu.2017.6746

Ibáñez, J. (2017) Horizontes para los educadores. Las promociones educativas y la promoción de la plenitud humana. Madrid, Dikynson.

Kong, S.C. (2014) «Developing information literacy and critical thinking skills through domain knowledge learning in digital classrooms: An experience of practicing flipped classroom strategy». Computers \& Education (Ámsterdam, Elsevier B.V.), 78, pp. 160-173. doi: https://doi.org/10.1016/j.compedu.2014.05.009

Larrosa, F. (2010) «Vocación docente versus profesión docente en las organizaciones educativas». Revista Electrónica Interuniversitaria de Formación del Profesorado (Murcia, Universidad de Murcia), 13(4), pp. 43-51.

Lasky, S. (2005) «A sociocultural approach to understanding teacher identity, agency and professional vulnerability in a context of secondary school reform». Teaching and Teacher Education (Ámsterdam, Elsevier B.V.), 21, pp. 899-916.

Loader, B., Vromer, A. y Xenos, M. (2014) «The networked Young citizen: social media, political participation and civic engagment». Information, 
Communication \& Society (United Kingdom, Taylor \& Francis, Ltd.), 17(2), pp. 143-150. doi: https://doi.org/10.1080/1369118X.2013.871571

López, E. (2016) «La formación docente del profesorado universitario: sentido, contenido y modalidades». Bordón (Madrid, Sociedad Española de Pedagogía), 68(4), pp. 89-102. doi: https://doi.org/10.13042/Bordon.2016.38998

Madrid, D. y Mayorga, M.J. (2017) «Construcción y reconstrucción de la identidad de los futuros docentes a través de sus autobiografías académicas». Revista Complutense de Educación (Madrid, Universidad Complutense de Madrid), 28(2), pp. 375-389.

Marcelo, C. (2010) «La identidad docente: constantes y desafíos». Revista Interamericana de Investigación, Educación y Pedagogía (Bogotá, Universidad Santo Tomás), 3(1), pp. 15-42. doi: https://doi.org/10.15332/s1657107X.2010.0001.01

Martín, Á., Conde, J. y Mayor, C. (2014) «La identidad profesional docente del profesorado novel universitario». REDU. Revista de Docencia Universitaria (Valencia, Universitat Politècnica de València), 12(4), pp. 141-160. doi: https://doi.org/10.4995/redu.2014.5618

Martínez, M., Esteban, F., Gonzalo, J. y Payá, M. (2016) La educación, en teoría. Madrid, Síntesis.

Matsuba, M.K., Murzyn, T., y Hart, D. (2011) «A model of moral identity: Applications for education». Advances in Child Development and Behavior (Ámsterdam, Elsevier B.V.), 40, pp. 181-207.

Merino, D. (2002) Concepciones de los profesores y autoconcepto y agresividad de los alumnos en un contexto de educación intercultural. Málaga, Universidad de Málaga (tesis doctoral; director: José Manuel Esteve Zarazaga).

Mojarro, A., Rodrigo, D. y Etchegaray, M.C. (2015) «Educación personalizada a través de e-learning». Alteridad (Quito, Universidad Politécnica Salesiana de Ecuador), 10(1), pp. 21-30. doi: https://doi.org/ 10.17163/alt.v10n1.2015.02

Mora, F. (2013) Neuroeducación. Solo se puede aprender aquello que se ama. Madrid, Alianza Editorial.

Motos, P. y Aguilar, Á.I. (2018) «\#DíalnternacionaldelPuebloGitano: una mirada a la realidad educativa de la etnia gitana a través de Twitter». Innoeduca. International Journal of Technology and Educational Innovation (Málaga, Universidad de Málaga), 4(2), pp. 106-113.

doi: http://dx.doi.org/10.24310/innoeduca.2018.v4i2.4979

Noddings, N. (2010) "Moral education and caring». Theory and Research in Education (New York, SAGE Journals), 8(2), pp. 145-151.

Nussbaum, M. (2001) El cultivo de la humanidad. Barcelona, Editorial Andrés Bello.

Olven, B. (2016) Teaching for Success. Developing Your Teacher Identity in Today's Classroom. New York, Routledge.

Ordine, N. (2013) La utilidad de lo inútil. Manifiesto. Barcelona, Acantilado.

Pennington, M.C. y Richards, J.C. (2016) «Teacher Identity in Language Teaching: Integrating Personal, Contextual, and Professional Factors». RELC Journal (New York, SAGE Journals), 47(1), pp. 5-23.

doi: https://doi.org/10.1177/0033688216631219

Pérez, V. y Rodríguez, J.C. (2013) «Educación y prestigio docente en España: la visión de la sociedad». En Esteban, M. [coord.]. El prestigio de la profesión docente en España. Percepción y realidad. Informe. Madrid, Fundación Botín y Fundación Europea Sociedad y Educación, pp. 33-108. 
Quevedo, E. y Arruti, M.A. (2018) «El nuevo rol del docente como facilitador del aprendizaje». En Villa, A. [ed.]. Tendencias actuales de las transformaciones de las universidades en una nueva sociedad digital. Bilbao, Foro Internacional de Innovación Universitaria, pp. 195-208.

Recalcati, M. (2016) La hora de clase. Por una erótica de la enseñanza. Barcelona, Anagrama.

Rodríguez, M., González, A.M. y Gutiérrez, M.A. (2019) «Experiencias de la profesión docente: la construcción de la identidad y vocación». RECIE. Revista Electrónica Científica de Investigación Educativa (Chihuahua, Red de Investigadores Educativos Chihuahua AC), 4(2), pp. 1317-1325.

Romero, C. y Núñez, L. (2017) «Reformular la práctica a través de las filosofías educativas». En Núñez, L. y Romero, C. [coords.]. Teoría de la educación. Capacitar para la práctica. Madrid, Pirámide, pp. 35-55.

Romero, E. y Robinson, N. (2017) «Laboratorios sociales en Universidades: Innovación e impacto en Medialab UGR». Comunicar (Huelva, Grupo Comunicar), 51, pp. 29-38. doi: https://doi.org/10.3916/C51-2017-03

Ruiz, C. (2005) Identidades transculturales: los procesos de construcción de identidad de los hijos de inmigrantes marroquíes en España. Málaga, Universidad de Málaga (tesis doctoral; director: José Manuel Esteve Zarazaga).

Sanderse, W. (2013) «The meaning of role modelling in moral and character education». Journal of Moral Education (United Kingdom, Taylor \& Francis, Ltd.), 42(1), pp. 28-42.

Scott, G.A. (2000) Plato's Socrates as Educator. New York, SUNY Press.

Serret, A., Martí, M. y Corbatón, R. (2016) «Las historias de vida en la formación de maestros de educación infantil». Revista Iberoamericana de Educación (Madrid, Organización de Estados Iberoamericanos), 71, pp. 217-228.

Tello, C. (2016) «Políticas de subjetividad: autonomía e identidad docente en Latinoamérica». Interacções (Santarém, Escola Superior de Educação de Santarém), 40(12), pp. 70-88.

Timmerman, G. (2009) «Teacher educators modelling their teachers?» European Journal of Teacher Education (United Kingdom, Taylor \& Francis, Ltd.), 32(3), pp. 225-238.

Unamuno, M. (1917) Comentario. Madrid, Boletín de la Institución Libre de Enseñanza.

UNESCO (2018) Docentes. Recuperado de https://bit.ly/2hkkTZt [acceso: 19.11.2018].

Valle, J.M. (2013) «¿Hay que cambiar la formación inicial de los docentes para elevar el prestigio social de maestros y profesores?» En Esteban, M. [coord.]. El prestigio de la profesión docente en España. Percepción y realidad. Informe. Madrid, Fundación Botín y Fundación Europea Sociedad y Educación, pp. 138-141. 
Resum: En un context sociocultural dinàmic i flexible, les funcions i competències que s'associen als docents es van adaptant a les demandes i necessitats que es generen a la societat. Per tal de conèixer com és el mestre que la societat valora actualment, aquest estudi analitza la figura del docent a través de la xarxa social Twitter a partir del hashtag \#DíaMundialdelDocente. La mostra està formada per 1441 tweets que van ser sotmesos a una anàlisi en dues fases: una classificació dels missatges per temàtica i una posterior categorització en funció dels arquetips de mestres. Els resultats reflecteixen que la temàtica amb més missatges registrats ha estat la reivindicativa, mentre que l'arquetip més valorat a nivell social és el del mestre autèntic i personal. En les conclusions sobresurt el reconeixement social positiu que existeix sobre la figura del mestre, així com la possibilitat d'incorporar les qualitats i competències que són valorades per la societat als processos de formació inicial i contínua del professorat.

Paraules clau: Característiques del mestre, rol del mestre, xarxes socials, opinió pública, Twitter, influència de la tecnologia.

\section{La perception sociale des enseignants : une analyse de la figure de l'enseignant à travers Twitter}

Résumé: Dans un contexte socioculturel dynamique et flexible, les fonctions et les compétences qui sont associées aux enseignants s'adaptent aux attentes et aux besoins qui naissent dans la société. Afin de découvrir la figure de l'enseignant valorisée actuellement par la société, nous procédons à son analyse à travers le réseau social Twitter à partir de l'hashtag \#DíaMundialdelDocente (\#Journeemondialedelenseignant). L'échantillon est composé de 1441 tweets qui ont été soumis à une analyse réalisée en deux phases: les messages ont d'abord été classés par thématique, puis catégorisés en fonction des archétypes d'enseignants. D'après les résultats obtenus, la thématique à caractère revendicateur est celle qui a enregistré le plus de messages, tandis que l'archétype le plus valorisé sur le plan social est celui de l'enseignant authentique et personnel. Les conclusions mettent en valeur la reconnaissance sociale positive à l'égard de la figure de l'enseignant, ainsi que la possibilité d'inclure les qualités et les compétences qui sont valorisées par la société dans les processus de formation initiale et continue du corps enseignant.

Mots clés: Caractéristiques de l'enseignant, rôle de l'enseignant, réseaux sociaux, opinion publique, Twitter, influence de la technologie.

\section{Through society's eyes: an analysis of the figure of the teacher using Twitter}

Abstract: In our dynamic and protean sociocultural environment, teachers must tailor their roles and competences to society's needs. To determine how teachers are currently valued by society, this study analyses data drawn from the social networking service Twitter, from the hashtag \#DíaMundialdelDocente. The sample consisted of 1,441 tweets analysed in two phases. First, the tweets were classified according to the subject they discussed, and then they were also grouped under specific teacher archetypes. The results show that the most popular subject was some form of critique of the education system, while the most highly-valued archetype was the teacher with a sincere and personable manner. The article concludes that teachers receive positive social recognition and affirms that it is possible to incorporate the qualities and competencies that society values in the processes of initial and in-service teacher education.

Keywords: Teacher characteristics, teacher's role, social networks, public opinion, Twitter, influence of technology. 